\title{
Determination of fosfomycin susceptibility in carbapenemase- producing $K$. pneumoniae strains isolated prior to clinical use of the intravenous formulation in Turkey
}

\author{
Gülsen ALTINKANAT GELMEZ ${ }^{1}$ (D), Baris CAN $^{3}$ (D), Buket ERTURK SENGEL ${ }^{2}$ (D), Volkan KORTEN ${ }^{2}$ (D), Guner SOYLETIR ${ }^{1}$ (iD
}

${ }^{1}$ Department of Medical Microbiology, School of Medicine, Marmara University, Istanbul, Turkey.

${ }^{2}$ Department of Infectious Diseases, School of Medicine, Marmara University, Istanbul, Turkey.

${ }^{3}$ Department of Medical Microbiology, Pendik Training and Research Hospital, Marmara University, Istanbul, Turkey.

\section{Corresponding Author: Gulsen ALTINKANAT GELMEZ}

E-mail: gulsenaltinkanat@yahoo.com

Submitted: 09.02.2021 Accepted: 12.04.2021

\section{ABSTRACT}

Objectives: The incidence of infections caused by carbapenem-resistant Enterobacteriaceae has increased worldwide. Limitations in the development of new antimicrobial agents have led clinicians to reconsider the clinical efficiency of old antibiotics, such as intravenous formulation of fosfomycin, in the treatment of multidrug-resistant Gram-negative bacterial infections. We investigated the fosfomycin susceptibility of carbapenemase-producing Klebsiella pneumoniae strains isolated prior to the clinical use of the intravenous formulation of fosfomycin in Turkey.

Materials and Methods: A total of the $155 \mathrm{~K}$. pneumoniae isolates which previously characterized at the molecular level for their carbapenemase were included in this study. The minimum inhibitory concentration of fosfomycin was determined by the agar dilution method.

Results: Overall, $65.1 \%$ of the isolates were susceptible to fosfomycin. The MIC50 and MIC 90 values were 32 and $256 \mathrm{mg} / \mathrm{L}$, respectively. According to our results, at least two-third of carbapenemase-positive K. pneumoniae are susceptible to fosfomycin.

Conclusions: Although, the susceptibility of fosfomycin, which has just been put into the clinical use of intravenous formulation in Turkey, is not very high, it can be considered as an alternative adjunct antimicrobial for the treatment of systemic infections caused by carbapenemase-positive K. pneumoniae isolates.

Keywords: Fosfomycin susceptibility, Carbapenemase-producing Klebsiella pneumoniae

\section{INTRODUCTION}

The emergence and spread of carbapenem-resistant Enterobacteriaceae (CRE) have become a global health problem during the last decade. Generally, CRE isolates are multi-drugresistant, and the most prevalent mechanisms of carbapenem resistance in Enterobacteriaceae have been associated with the production of carbapenem-hydrolyzing enzymes. Different types of class A (e.g. KPC, GES), class B (e.g. NDM, IMP, VIM) and class D (e.g. OXA-48-like) enzymes have been identified in Enterobacteriaceae [1-3]. Treatment options of infections caused by CRE are challenging due to limited antimicrobials available. Therefore, it is very important to develop new and effective therapeutic strategies [4].
The limitations in the development of new antimicrobial agents have led physicians to reassess the clinical efficacy of old antibiotics, such as fosfomycin, in the treatment of multidrugresistant Gram-negative bacterial infections. Fosfomycin is an old broad-spectrum antibiotic and active against both Grampositive and Gram-negative bacteria [5]. Oral fosfomycin is commonly used for the treatment of uncomplicated urinary tract infections. The intravenous formulation has been available in many European countries (United Kingdom, Spain, France, Germany, Austria, Greece, etc.) and some Asian countries (Japan) for the treatment of systemic infections for several years [6]. In some European studies, the use of IV fosfomycin

How to cite this article: Gelmez Altinkanat G, Can B, Sengel Erturk B, Korten V, Soyletir G. Determination of fosfomycin susceptibility in carbapenemaseproducing K. pneumoniae strains isolated prior to clinical use of the intravenous formulation in Turkey. Marmara Med J 2021; 34(2):127-131. doi: 10.5472/marumj.942784 
had a good effect on clinical and microbiological outcome in patients infected with various carbapenem-resistant Klebsiella pneumoniae strains. Fosfomycin seems to be an alternative agent for the treatment of such difficult to treat infections [7]. However, one of the main problems with this drug is the potential emergence of resistance during monotherapy. Therefore, it is recommended to use fosfomycin in combination with other antimicrobial agents (such as carbapenems, aminoglycosides, colistin, and tigecycline) in serious infections. Although, various combination regimens are used for the treatment of CRE infections, the clinical evidence is mainly based on case reports and retrospective studies. Therefore, more studies are needed to determine fosfomycin efficiency in the treatment of systemic infections $[4,6]$.

The intravenous formulation of fosfomycin was introduced in December 2018 for the treatment of systemic infections in Turkey. In this study, we investigated the fosfomycin susceptibility of carbapenemase-producing K. pneumoniae strains isolated prior to the commercial availability of the intravenous formulation of fosfomycin.

\section{MATERIALS and METHODS}

\section{Study isolates}

The Klebsiella pneumoniae isolates used in this study were collected from various clinical samples (bronchoalveolar lavage, deep tracheal aspirate, sputum, blood, urine, wound) of infected patients between January 2015 and December 2017 from hospitalized patients at Marmara University Hospital, Istanbul, Turkey. All of the non-duplicate isolates were identified by matrix-assisted laser desorption ionization-time of flight mass spectrometry (VITEK-MS, bioMerieux, Marcy l'Etoile, France). Carbapenem susceptibility was initially determined using an automated antimicrobial susceptibility test system (VITEK2, bioMerieux, Marcy-l'E'toile, France). Minimal inhibitory concentrations (MICs) of imipenem, meropenem, and ertapenem were determined by the gradient diffusion strip test (Etest ${ }^{\oplus}$, bioMérieux, Marcy l'Etoile, France) and interpreted according to European Committee on Antimicrobial Susceptibility Testing (EUCAST) guidelines [8]. Molecular detection of the different carbapenemase genes was carried out by in-house polymerase chain reaction (PCR) using the specific primers for genes encoding $b l a_{\mathrm{VIM}}, b l a_{\mathrm{IMP}}, b l a_{\mathrm{NDM}}, b l a_{\mathrm{KPC}}$, and $b l a_{\text {OXA-48 }}$ [9-12]. A total of 155 carbapenemase-positive $K$. pneumoniae isolates confirmed by polymerase chain reaction (PCR) method were selected for fosfomycin susceptibility testing. We excluded isolates carrying more than one type of carbapenemases.

\section{Fosfomycin susceptibility testing}

Fosfomycin trometamol was obtained from Drogsan (Istanbul, Turkey) and glucose-6-phosphate (G6P) from Sigma Aldrich ((St. Louis, MO, USA). The agar dilution method was used to determine the susceptibility of fosfomycin on Mueller Hinton agar (MHA) ((Becton Dickinson, New Jersey, USA) supplemented with $25 \mathrm{mg} / \mathrm{L}$ G6P, according to the EUCAST guidelines. The MHA plates containing fosfomycin in concentrations from 0.06 to $256 \mathrm{mg} / \mathrm{L}$ were prepared, surfaceinoculated with $10^{4} \mathrm{CFU} / \mathrm{mL}$ and allowed to dry. The plates were incubated for 16 to $20 \mathrm{~h}$ at ambient air at $35^{\circ} \mathrm{C}$. Results of antibiotic susceptibility testing were interpreted using the EUCAST criteria, where $\leq 32 \mathrm{mg} / \mathrm{L}$ is considered as susceptible and $>32 \mathrm{mg} / \mathrm{L}$ as resistant [8]. Escherichia coli ATCC 25922 was used as the quality control strain.

\section{Statistical Analysis}

The differences in fosfomycin susceptibility between the groups were compared using the Fisher's exact test. The differences between the groups were considered significant if the $\mathrm{p}$-values were smaller than 0.05 .

\section{RESULTS}

Carbapenemase-positive isolates expressed OXA-48 ( $\mathrm{n}=$ 82), NDM ( $\mathrm{n}=50)$, IMP ( $\mathrm{n}=11), \operatorname{KPC}(\mathrm{n}=7)$, and VIM ( $\mathrm{n}$ $=5$ ) carbapenemase. The isolates were resistant at least one of the carbapenems (ertapenem, imipenem, and meropenem) according to the EUCAST guidelines.

The fosfomycin MIC range was determined between $2 \mathrm{mg} / \mathrm{L}$ and $>256 \mathrm{mg} / \mathrm{L}$ using agar dilution method. According to the EUCAST criteria, $65.1 \%$ of the isolates were susceptible to fosfomycin. While the highest fosfomycin susceptibility was detected in KPC-positive isolates, the lowest susceptibility was found in IMP-positive isolates (Table I). The $\mathrm{MIC}_{50}$ and $\mathrm{MIC}_{90}$ values were 32 and $256 \mathrm{mg} / \mathrm{L}$, respectively. Among the various carbapenemase types, KPC-positive isolates had the lowest $\mathrm{MIC}_{50}$ and $\mathrm{MIC}_{90}$ values (Table I). 
Table I. Fosfomycin susceptibility in various types of carbapenemase producing K. pneumoniae

\begin{tabular}{|c|c|c|c|c|c|c|c|c|c|c|c|c|c|}
\hline \multirow[t]{2}{*}{ Carbapenemase type } & \multicolumn{5}{|c|}{ Susceptible (n) } & \multicolumn{3}{|c|}{ Resistant (n) } & \multirow[t]{2}{*}{$\mathrm{MIC}_{50}(\mathrm{mg} / \mathrm{L})$} & \multirow[t]{2}{*}{$\mathrm{MIC}_{90}(\mathrm{mg} / \mathrm{L})$} & \multirow{2}{*}{$\begin{array}{l}\text { MIC Range } \\
(\mathrm{mg} / \mathrm{L})\end{array}$} & \multirow{2}{*}{$\begin{array}{c}\text { Fosfomycin } \\
\text { susceptibility } \\
(\%)\end{array}$} & \multirow[t]{2}{*}{ p } \\
\hline & $2^{\star}$ & $4^{*}$ & $8^{*}$ & $16^{*}$ & $32^{*}$ & $64^{*}$ & $128^{*}$ & $256^{*}$ & & & & & \\
\hline OXA-48-like $(\mathrm{n}=82)$ & 1 & & 13 & 14 & 26 & 12 & 3 & 13 & 32 & 256 & $2->256$ & 65.8 & 1 \\
\hline $\operatorname{NDM}(n=50)$ & & 1 & 2 & 14 & 16 & 6 & 5 & 6 & 32 & 256 & $4->256$ & 66 & 1 \\
\hline $\operatorname{IMP}(n=11)$ & & 1 & 1 & 1 & 3 & 2 & 2 & 1 & 32 & 128 & $4->256$ & 54.5 & 1 \\
\hline $\operatorname{KPC}(\mathbf{n}=7)$ & 1 & & 2 & 1 & 1 & 1 & & 1 & 8 & 64 & $2-256$ & 71.4 & 0,52 \\
\hline $\operatorname{VIM}(n=5)$ & & & 3 & & & & 1 & 1 & 8 & 128 & $8-256$ & 60 & 1 \\
\hline TOTAL $(\mathbf{n}=155)$ & 2 & 2 & 21 & 30 & 46 & 21 & 11 & 22 & 32 & 256 & $2->256$ & 65.1 & \\
\hline
\end{tabular}

MIC: Minimal inhibitory concentration $(m g / L)$

* The MICs given in the table are the susceptibility breakpoint for fosfomycin recommended by EUCAST.

\section{DISCUSSION}

Antimicrobial resistance in Gram-negative microorganisms is a global problem. In particular, carbapenem-resistant $K$. pneumoniae has spread all over the world in the last decade and represents one of the most critical challenges to the antimicrobial therapy of such infections. Because of the shortage of new antimicrobials, clinicians have begun to re-evaluate already existing antibiotics such as fosfomycin.

Fosfomycin is a small, hydrophilic agent with almost negligible serum protein binding. It is a broad-spectrum agent that is active against multidrug-resistant bacterial pathogens (MRSA, VRE, ESBL, CRE) and is excreted unchanged in the urine, reaching high urinary concentrations after administration of the 3-g single dose [13]. Therefore, the oral formulation is generally safe in uncomplicated urinary tract infections. Rapid penetration and distribution of fosfomycin into tissues such as soft tissue, lungs, bone, and cerebrospinal fluid is an important feature for preference in the treatment of serious infections caused by MDR-pathogens [7]. While intravenous fosfomycin has been prescribed for a wide variety of infections in many countries for more than 40 years, this formulation has been introduced into Turkey in late 2018. Therefore, clinical and in-vitro susceptibility studies are limited in this country $[14,15]$. Fosfomycin susceptibility among CRE ranges between 42 and 98\%, according to results of studies conducted in different countries [16-25]. We found a $65.1 \%$ susceptibility rate of fosfomycin against 155 carbapenemase-positive Klebsiella pneumoniae isolates in our study. Fosfomycin susceptibility rate found in our study was lower when compared to previous studies, mainly because most of these studies were performed only on KPC-producing strains and were evaluated according to EUCAST criteria (susceptible $\leq$ $64 \mathrm{mg} / \mathrm{L}$ ) before 2011. Susceptibility data of CRE carrying other carbapenemases is also limited. Our study results will contribute to the literature in account of having the results for the most frequently observed five carbapenemase types (OXA-48, NDM, KPC, IMP, VIM) and evaluating them according to the current EUCAST fosfomycin breakpoint.

After intravenous administration of fosfomycin, mean peak serum concentrations commonly range from 200 to 600 $\mathrm{mg} / \mathrm{L}$, according to several studies [13]. These values are well above the clinical breakpoints determined by EUCAST for Enterobacteriaceae (susceptible $\leq 32 \mathrm{mg} / \mathrm{L}$ ). Also, fosfomycin MICs of $85.8 \%$ of our study isolates had $\leq 128 \mathrm{mg} / \mathrm{L}$ and were below the achievable serum concentration values.

The correlations of PD parameters (concentration and/or timedependent activity) with antibacterial activity of fosfomycin have not yet been clarified. Although it is known that even high doses (20-24 g/day) are well-tolerated, no definitive treatment protocol has been established yet. However, it should be remembered that resistance may develop during the use of fosfomycin monotherapy, and resistance development has been reported in approximately $3.4 \%$ during the treatment in some studies $[6,25]$. Therefore, in serious infections, it is recommended to use it in combination with other antimicrobial agents such as carbapenems, aminoglycosides, colistin or 
tigecycline [26, 27]. In some studies, treatment success rates of $>80 \%$ have been achieved with the combined use of fosfomycin with other antimicrobial agents in the treatment of serious infections [6, 28-31].

The limitation of this study is that excluded isolates carrying more than one type of carbapenemases. Those isolates may have a different susceptibility pattern.

In the light of these findings, intravenous fosfomycin administration may be a valuable option in the treatment of CRE infections. For this reason, the determination of fosfomycin susceptibility in CRE strains has become a crucial issue in clinical microbiology laboratories, since the EUCAST recommends the agar dilution method for Enterobacteriaceae, which is not suitable for use in routine laboratory [8].

According to our study results, at least two-third of carbapenemase-positive K. pneumoniae in our center were susceptible to fosfomycin before the availability of the intravenous formulation, and it seems to be used as an alternative adjunct antimicrobial for the treatment of systemic infections caused by carbapenemase-positive $K$. pneumoniae isolates. It, therefore, seems adequate that laboratories should routinely perform fosfomycin susceptibility testing with reference methods to guide clinicians appropriately.

\section{Compliance with Ethical Standards}

Ethical approval: The study protocol was approved by the Marmara University, School of Medicine Ethics Committee ( approval number: 09.2018.831).

Conflict of interest: The authors declare that they have no conflict of interest.

Financial Support: The authors have no relevant financial information to disclose.

Authors' contributions: Concept, study design and conduct: GAG, GS and VK, Data acquisition: GAG, BES and BC, Data analysis and interpretation: GAG, BES and BC, GAGG. Altinkanat Gelmez, B. Erturk Sengel and B. Can. Drafting: GAG. All authors critically reviewed the manuscript and approved the final version.

\section{REFERENCES}

[1] Tzouvelekis LS, Markogiannakis A, Psichogiou M, Tassios PT, Daikos GL. Carbapenemases in Klebsiella pneumoniae and other Enterobacteriaceae: an evolving crisis of global dimensions. Clin Microbiol Rev 2012; 25:682-707. doi: 10.1128/CMR.05035-11

[2] Queenan AM, Bush K. Carbapenemases: the versatile betalactamases. Clin Microbiol Rev 2007; 20(3):440-458. doi: 10.1128/CMR.00001-07

[3] Walsh TR. Emerging carbapenemases: a global perspective. Int J Antimicrob Agents 2010; 36 Suppl 3: 8-14. doi: 10.1016/ S0924-8579(10)70004-2

[4] Sheu CC, Chang YT, Lin SY, Chen YH, Hsueh PR. Infections caused by carbapenem-resistant Enterobacteriaceae: An update on therapeutic options. Front Microbiol 2019; 10:80. doi: $10.3389 /$ fmicb. 2019.00080

[5] Raz R. Fosfomycin: an old-new antibiotic. Clin Microbiol Infect 2012;18(1):4-7. doi: 10.1111/j.1469-0691.2011.03636.x

[6] Grabein B, Graninger W, Rodríguez Baño J, et al. Intravenous fosfomycin-back to the future. Systemic review and metaanalysis of the clinical literature. Clin Microbiol Infect 2017; 23(6): 363-372. doi: 10.1016/j.cmi.2016.12.005

[7] Michalopoulos A, Virtzili S, Rafailidis P, et al. Intravenous fosfomycin for the treatment of nosocomial infections caused by carbapenem-resistant Klebsiella pneumoniae in critically ill patients: a prospective evaluation. Clin Microbiol Infect 2010; 16(2):184-186. doi: 10.1111/j.1469-0691.2009.02921.x

[8] European Committee on Antimicrobial Susceptibility Testing (EUCAST): Clinical breakpoints version 9.0. In: European Committee on Antimicrobial Susceptibility Testing. Vaxjo: EUCAST 2019. Available from: http://www.eucast.org

[9] Aktaş Z, Kayacan CB, Schneider I, et al. Carbapenem hydrolyzing oxacillinase, OXA-48, persists in Klebsiella pneumoniae in Istanbul, Turkey. Chemotherapy 2018; 54(2):101-106. doi: 10.1159/000118661.

[10] Perry JD, NaqviSH, Mirza IA, et al. Prevalence of faecal carriage of Enterobacteriaceae with NDM-1 carbapenemase at military hospitals in Pakistan, and evaluation of two chromogenic media. J Antimicrob Chemother 2011; 66(10):2288-2294. doi: 10.1093/jac/dkr299

[11] Pitout JD, Gregson DB, Poirel L, et al._Detection of Pseudomonas aeruginosa producing metallo-beta-lactamases in a large centralized laboratory. J Clin Microbiol 2005; 43(7):3129-35. doi: 10.1128/JCM.43.7.3129-3135.2005.

[12] Cole JM, Schuetz AN, Hill CE, Nolte FS. Development and evaluation of a real-time PCR assay for detection of Klebsiella pneumoniae carbapenemase genes. J Clin Microbiol 2009; 47(2):322-326. doi: 10.1128/JCM.01550-08

[13] Roussos N, Karageorgopoulos DE, Samonis G, Falagas ME. Clinical significance of the pharmacokinetic and pharmacodynamic characteristics of fosfomycin for the treatment of patients with systemic infections. Int J Antimicrob Agents 2009; 34(6):506-515. doi: 10.1016/j. ijantimicag.2009.08.013

[14] Evren E, Azap KO, Çolakoğlu Ş, Arslan H. In vitro activity of fosfomycin in combination with imipenem, meropenem, colistin and tigecycline against OXA 48-positive Klebsiella pneumoniae strains. Diagn Microbiol Infect Dis 2013;76(3):335-8. doi: 10.1016/j.diagmicrobio.2013.04.004

[15] Yıldız SS, Kaşkatepe B, Şimşek H, Sarıüzel FM. High rate of colistin and fosfomycin resistance among carbapenemaseproducing Enterobacteriaceae in Turkey. Acta Microbiol Immunol Hung, 2019 Mar 1;66(1):103-112. doi: 10.1556/030.65.2018.042.

[16] Livermore DM, Warner M, Mushtaq S, et al. What remains against carbapenem-resistant Enterobacteriaceae? Evaluation of chloramphenicol, ciprofloxacin, colistin, fosfomycin, minocycline, nitrofurantoin, temocillin, and tigecycline. 
Int J Antimicrob Agents 2011; 37:415-419. doi: 10.1016/j. ijantimicag.2011.01.012.

[17] Bielen L, Likić R, Erdeljić V, et al. Activity of fosfomycin against nosocomial multiresistant bacterial pathogens from Croatia: a multicentric study. Croat Med J 2018;59(2):56-64. doi: $10.3325 / \mathrm{cmj} .2018 .59 .56$

[18] Kaase M, Szabados F, Anders A, Gatermann SG. Fosfomycin susceptibility in carbapenem-resistant Enterobacteriaceae from Germany. J Clin Microbiol 2014; 52:1893-1897. doi: 10.1128/JCM.03484-13

[19] Camarlinghi G, Parisio EM, Antonelli A, et al. Discrepancies in fosfomycin susceptibility testing of KPC-producing Klebsiella pneumoniae with various commercial methods. Diagn Microbiol Infect Dis 2019; 93(1):74-76. doi: 10.1016/j. diagmicrobio.2018.07.014

[20] Tuon FF, Rocha JL, Formighieri MS, et al. Fosfomycin susceptibility of isolates with $b l a_{\mathrm{KPC}-2}$ from Brazil. J Infect 2013; 67:247-249. doi: 10.1016/j.jinf.2013.04.017

[21] Kopacz J, Mariano N, Colon-Urban R, et al. Identification of extended-spectrum-beta-lactamase-positive Klebsiella pneumoniae urinary tract isolates harboring KPC and CTX-M beta-lactamases in nonhospitalized patients. Antimicrob Agents Chemother 2013; 57:5166-5169. doi: 10.1128/ AAC.00043-13

[22] Endimiani A, Patel G, Hujer KM, et al. In vitro activity of fosfomycin against bla ${ }_{\mathrm{KPC}}$ containing Klebsiella pneumoniae isolates, including those nonsusceptible to tigecycline and/ or colistin. Antimicrob Agents Chemother 2010; 54:526-529. doi: 10.1128/AAC.01235-09

[23] Souli M, Galani I, Antoniadou A, et al. An outbreak of infection due to beta-lactamase Klebsiella pneumoniae carbapenemase2-producing K. pneumoniae in a Greek university hospital: molecular characterization, epidemiology, and outcomes. Clin Infect Dis 2010; 50:364-373. doi: 10.1086/649865

[24] Paño-Pardo JR, Ruiz-Carrascoso G, Navarro-San Francisco $\mathrm{C}$, et al. Infections caused by OXA-48-producing Klebsiella pneumoniae in a tertiary hospital in Spain in the setting of a prolonged, hospital-wide outbreak. J Antimicrob Chemother 2013; 68:89-96. doi: 10.1093/jac/dks364

[25] Choudhury S, Yeng JL, Krishnan PU. In vitro susceptibilities of clinical isolates of carbapenemase-producing Enterobacteriaceae to fosfomycin and tigecycline. Clin Microbiol Infect 2015; 21(10): e75-6. doi: 10.1016/j. cmi.2015.06.005

[26] Karageorgopoulos DE, Miriagou V, Tzouvelekis LS, et al. Emergence of resistance to fosfomycin used as adjunct therapy in KPC Klebsiella pneumoniae bacteremia: report of three cases. J Antimicrob Chemother 2012; 67(11):2777-2779. doi: 10.1093/jac/dks270.

[27] Souli M, Galani I, Boukovalas S, et al. In-vitro interactions of antimicrobial combinations with fosfomycin against KPC-2 producing Klebsiella pneumoniae and protection of resistance development. Antimicrobial Agents Chemother 2011;55(5):2395-2397. doi: 10.1128/AAC.01086-10

[28] Karageorgopoulos DE, Wang $\mathrm{R}$, Yu XH, Falagas ME. Fosfomycin: evaluation of the published evidence on the emergence of antimicrobial resistance in Gram-negative pathogens. J Antimicrob Chemother 2012;67(2):255-268. doi: 10.1093/jac/dkr466.

[29] Falagas ME, Giannopoulou KP, Kokolakis GN, Rafailidis PI. Fosfomycin: use beyond urinary tract and gastrointestinal infections. Clin Infect Dis 2008; 46(7):1069-1077. doi: $10.1086 / 527442$.

[30] Falagas ME, Kastoris AC, Karageorgopoulos DE Rafailidis PI. Fosfomycin for the treatment of infections caused by multidrug-resistant non-fermenting Gram-negative bacilli: a systemic review of microbiological, animal and clinical studies. Int J Antimicrob Agents 2009;34(2): 111-120. doi: 10.1016/j.ijantimicag.2009.03.009.

[31] Falagas ME, Vouloumanou EK, Samonis G, Vardakas KZ. Fosfomycin. Clin Microbiol Rev 2016; 29 (2):21-347. doi: 10.1128/CMR.00068-15 